\title{
Histiocytose langerhansienne osseuse multifocale. À propos d'un cas
}

\section{RÉSUMÉ}

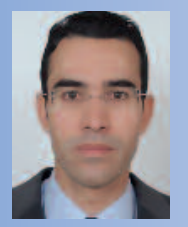

Hicham SABANI

Service de chirurgie plastique, chirurgie maxillo-faciale et stomatologie. Hôpital militaire d'instruction Mohamed V- Rabat.

Avenue des Far, Rabat, Maroc.

M. Karim EL KHATIB

Service de chirurgie plastique, chirurgie maxillo-faciale et stomatologie. Hôpital militaire d'instruction Mohamed V- Rabat.

\section{Jalal HAMAMA}

Service de chirurgie plastique, chirurgie maxillo-faciale et stomatologie. Hôpital militaire d'instruction Mohamed V- Rabat.

Mohammed NASSIH

Service de chirurgie plastique, chirurgie maxillo-faciale et stomatologie. Hôpital militaire d'instruction Mohamed V- Rabat.

Abdelkader RZIN

Service de chirurgie plastique, chirurgie maxillo-faciale et stomatologie. Hôpital militaire d'instruction Mohamed V- Rabat.

Youssef ZOUBIR

Service d'anatomo-pathologie. Hôpital militaire d'instruction Mohamed V- Rabat.

Mohammed OUKABLI

Service d'anatomo-pathologie. Hôpital militaire d'instruction

Mohamed V- Rabat.

Abderrahmane ALBOUZIDI

Service d'anatomo-pathologie. Hôpital militaire d'instruction Mohamed V- Rabat.
L'histiocytose langerhansienne est une maladie proliférative non maligne, qui intéresse les cellules dendritiques de Langerhans. II s'agit d'une maladie orpheline touchant essentiellement l'enfant et l'adulte jeune. Son étiologie reste encore inconnue, son spectre clinique est assez large.

Nous rapportons un cas clinique d'histiocytose langerhansienne osseuse multifocale chez un jeune adulte de 25 ans ayant touché initialement le rachis, et secondairement la mandibule et le crâne. Le diagnostic fut confirmé par l'examen histologique. L'évolution fut favorable après un traitement chirurgical non agressif.

À partir de cette observation, nous ferons une revue de la littérature pour mettre le point sur les aspects cliniques, histologiques, radiologiques, thérapeutiques et évolutifs de cette maladie orpheline. 


\section{Introduction}

$>$

L'histiocytose langerhansienne (HL), antérieurement connue sous le nom d'histiocytose $X$, peut être définie comme une maladie proliférative non maligne, qui intéresse les cellules dendritiques de Langerhans que l'on retrouve normalement au niveau des épidermes et dans les muqueuses situées à l'interface avec le milieu externe.

D’une façon générale, il s'agit d'une maladie orpheline avec une prévalence estimée à 2 cas pour 100000 [1], avec une certaine prédominance masculine, touchant essentiellement l'enfant et l'adulte jeune. Son étiologie reste encore inconnue en dépit des progrès réalisés

\section{Observation}

Nous rapportons une observation clinique $\mathrm{d}^{\prime} \mathrm{HL}$ osseuse multifocale chez un jeune adulte.

Il s'agit d'un jeune patient de 25 ans, tabagique, sans antécédent pathologique, hospitalisé en juillet 2007 au service de neurochirurgie pour prise en charge d'une HL osseuse localisée au niveau du rachis dorsolombaire, confirmée sur une biopsie osseuse du rachis, et pour laquelle il avait bénéficié d'un curetage suivi d'une ostéosynthèse D12-L2 pour prévenir une éventuelle fracture pathologique. L'évolution était marquée, six mois après, par une alvéolyse suivie de chute dentaire des dents $37,45,46$. Cette chute dentaire était indolore et sans cause traumatique, raison pour laquelle le patient nous a été adressé.

L'examen buccal révélait des lésions inflammatoires et nécrotiques très douloureuses des deux secteurs molaires inférieurs droit et gauche avec dans la compréhension de sa pathogénie. Son spectre clinique est assez large, néanmoins trois formes cliniques principales peuvent être dégagées [2] :

- la forme localisée : appelée également le granulome éosinophile, de l'adulte jeune, touchant surtout l'os et les poumons, et dont l'évolution est favorable ;

- la forme multifocale : de l'adolescent, c'est le granulome éosinophile multifocal d'évolution variable ;

- la forme pluritissulaire disséminée : du petit enfant, c'est la forme diffuse d'installation aiguë et dont l'évolution est sévère.

les dents 36, 44 et 47 qui étaient alvéolysées (fig. 1) et très mobiles, la peau en regard des lésions gingivales sus-décrites était normale et le signe de Vincent était négatif. Le reste de l'examen somatique était sans particularité.

Sur le plan biologique, il y avait un syndrome inflammatoire franc avec:

- une VS à $30 \mathrm{~mm}$;

- une CRP à 45,9 mg/L ;

- une $\alpha 2$ globuline à 10,4 g/L.

L'orthopantomogramme montrait deux images ostéolytiques bien limitées, la première sur la branche horizontale droite de la mandibule, englobant les racines des dents 44 et 47, la deuxième sur la branche horizontale gauche englobant la racine de la 36, sans signe de rhizalyse, réalisant l'aspect de dents flottantes ou de dents suspendues dans le vide (fig. 2). La radiographie du crâne révélait la présence 
d'une image lacunaire à l'emporte-pièce sans signe de condensation périphérique sur l'os frontal droit (fig. 3). La radiographie du rachis dorsolombaire montrait une réduction de la taille de l'image ostéolytique de D12 et L1.

Les radiographies des poumons, du bassin et des membres étaient toutes normales. Pour innocenter les organes abdominaux, en l'occurrence le foie et la rate, une échographie était réalisée et revenait sans anomalies.
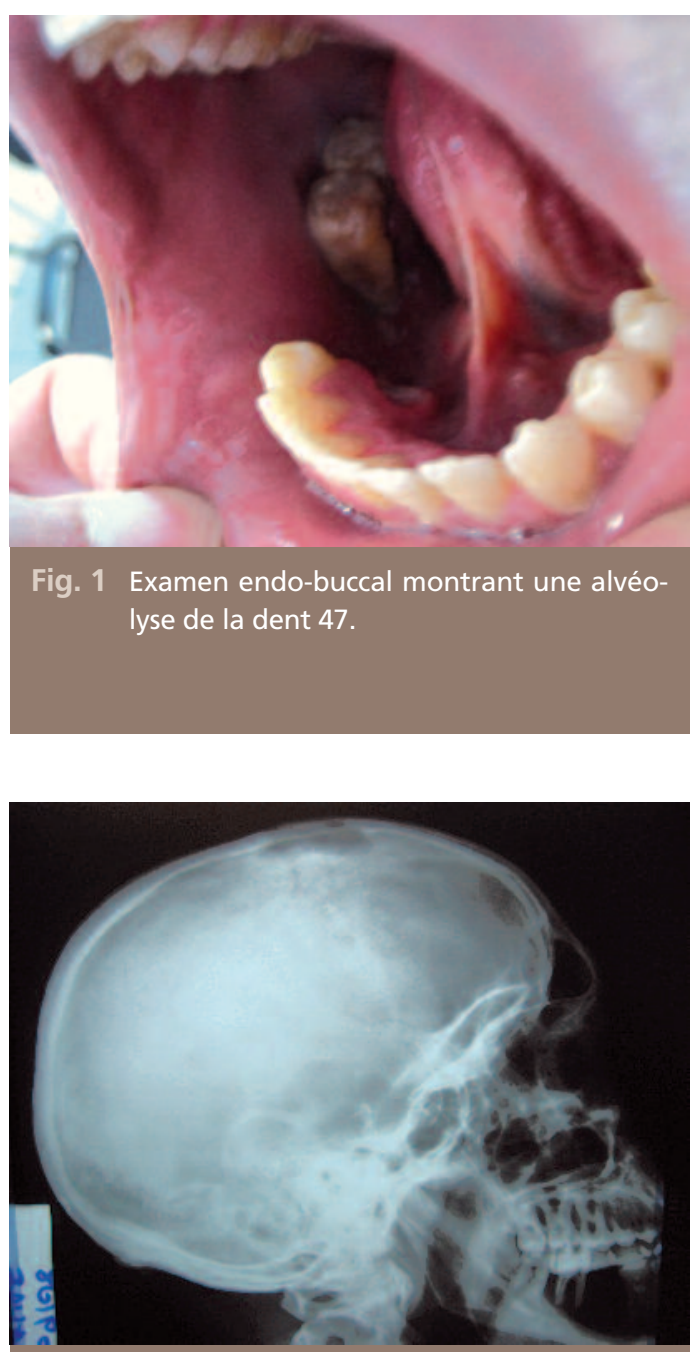

Fig. 3 Radiographie de crâne de profil montrant une image ostéolytique à l'emporte-pièce au niveau de l'os frontal droit.
La tomodensitométrie cranio-faciale montrait une énorme image lacunaire bien limitée rompant complètement l'os frontal droit et exerçant un effet de masse sur le parenchyme cérébral sous-jacent, limité par la dure-mère (fig. 4 à 6).

Pour confirmer qu'il s'agissait bien d'une poussée du granulome éosinophile localisé initialement au niveau du rachis dorsolombaire et pour écarter d'autres diagnostics pouvant prêter à confusion, en l'occurrence un myélome mul-

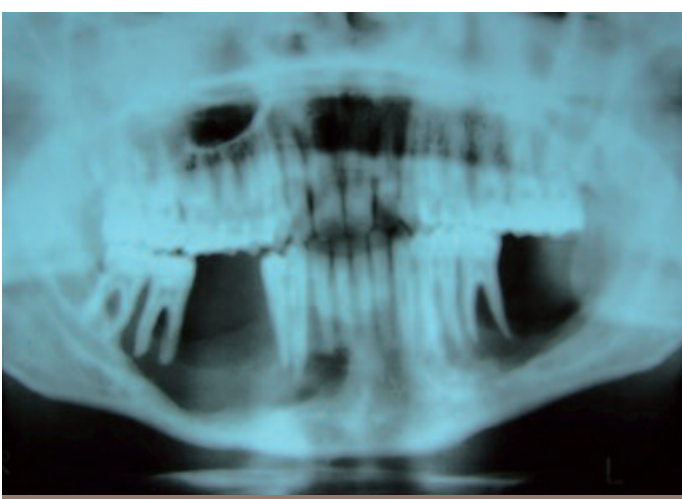

Fig. 2 Orthopantomogramme montrant des lésions ostéolytiques bien limitées des deux branches horizontales de la mandibule avec les dents $36,43,44$ et 47 « suspendues dans le vide $"$.

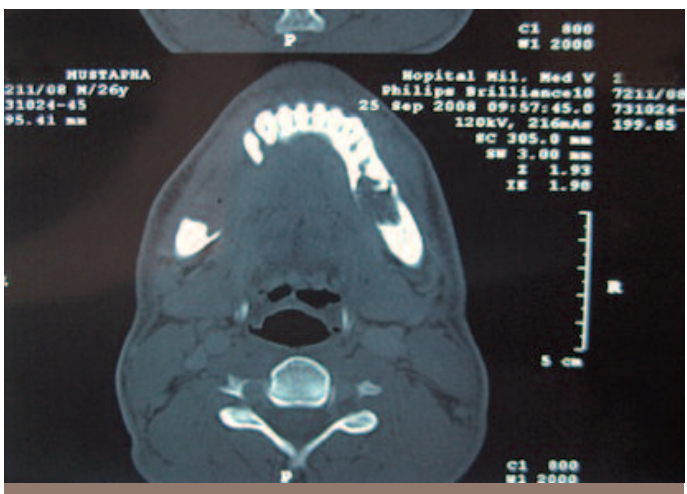

Fig. 4 Coupe axiale tomodensitométrique montrant des lésions ostéolytiques centromédullaires au niveau des deux branches horizontales de la mandibule avec rupture des deux tables interne et externe des deux côtés. 

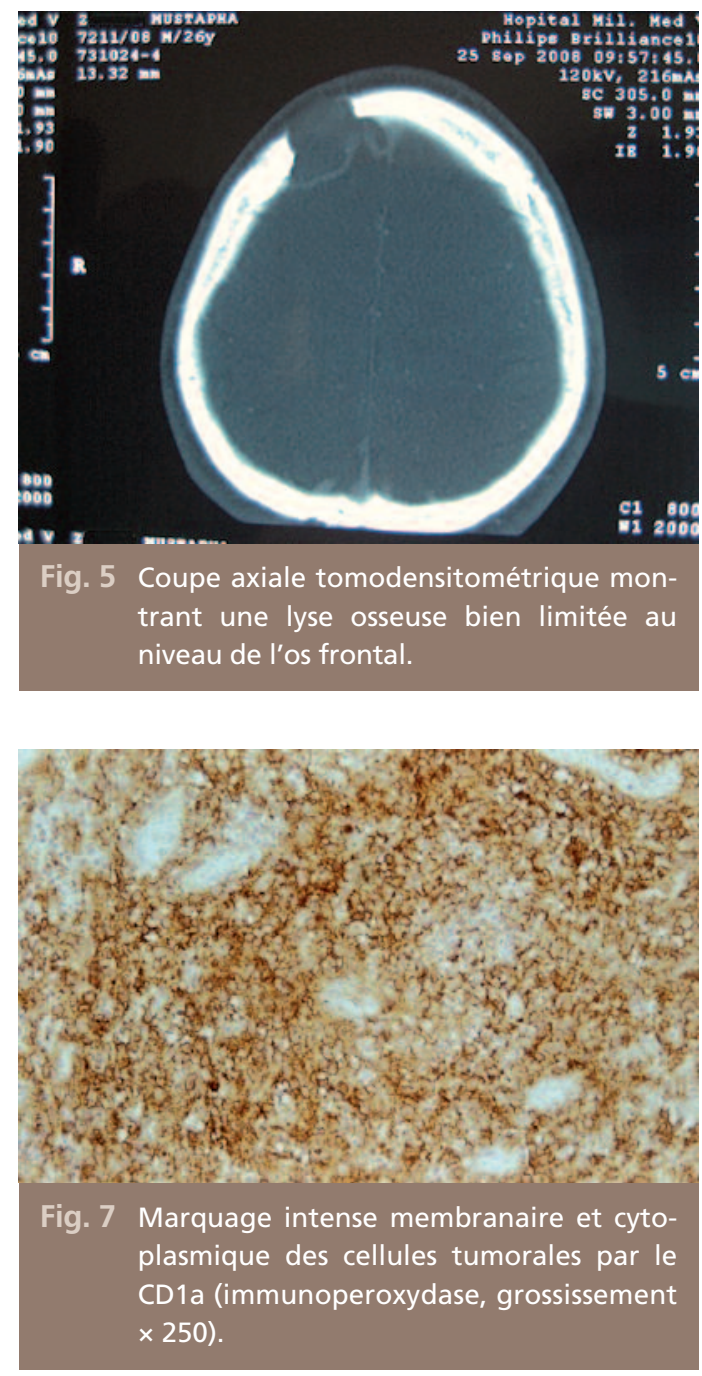

tiple ou des localisations osseuses secondaires d'une tumeur primitive, une biopsie osseuse mandibulaire sous anesthésie générale a été réalisée et montrait un aspect morphologique et immunohistochimique d'une HL (fig. 7 et 8). Le diagnostic d'HL fut ainsi retenu. Le patient a bénéficié d'un curetage appuyé des lésions

\section{Discussion}

En 1950, Lichtenstein regroupa sous le terme d'histiocytose trois entités cliniques diffé-

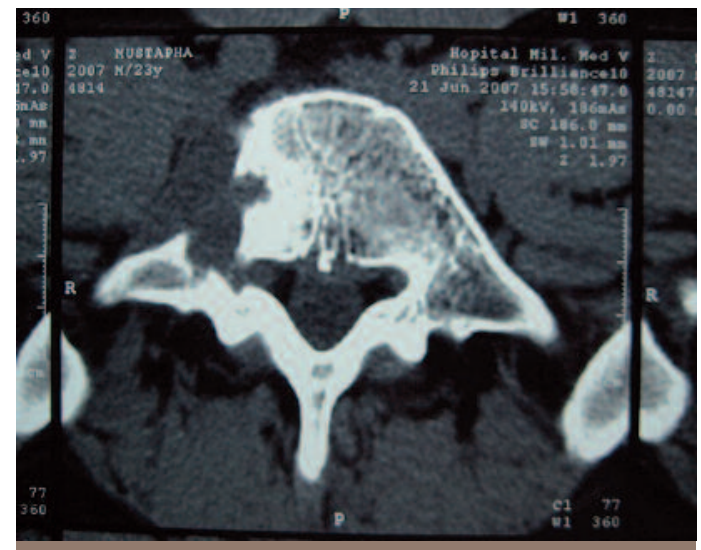

Fig. 6 Coupe axiale tomodensitométrique du rachis dorsolombaire montrant une lyse du corps vertébral.

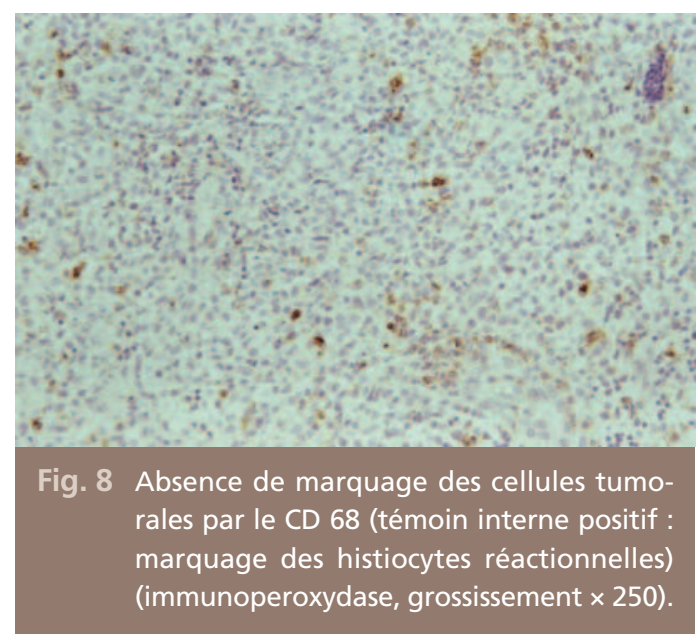

mandibulaires avec extraction des dents flottantes, avec un traitement médical symptomatique associant des antalgiques et des antiinflammatoires.

L'évolution fut favorable avec disparition progressive des douleurs et une bonne cicatrisation des lésions buccales.

rentes : le granulome éosinophile, le syndrome de Hand-Schuller-Christian et la maladie de 
Letterer-Siwe [3]. C'est en 1987 que l'Histiocyte Society Writing Group remplaça le terme d'histiocytose langerhansienne par celui d'histiocytose $X[4]$.

Le granulome éosinophile est la forme la plus fréquente de I'HL (50\%). II atteint essentiellement l'os dans $82 \%$ des cas. Il peut être unique (90\%) ou multiple (10\%), avec des atteintes simultanées ou successives $[5,6]$.

Alors que la forme unifocale peut survenir à tout âge, donnant des lésions osseuses isolées, la forme multifocale est particulièrement fréquente chez l'enfant et l'adulte jeune.

La localisation cranio-faciale est la plus fréquente (30-50 \% selon les séries), suivie par les localisations au rachis et aux os longs (10\% pour chacune). Une atteinte du bassin (surtout l'os iliaque) et des côtes existe, chacune dans $8 \%$ des cas [6]. L'observation que nous rapportons est celle d'un granulome éosinophile $d u$ rachis dorsolombaire qui a migré au niveau de la mandibule et du crâne.

Wilman et al. ont montré que l'HL est la conséquence d'une prolifération clonale de cellules de Langerhans pouvant être secondaire à la mutation somatique d'un gène [7]. Le caractère clonal de cette maladie n'est pas synonyme de malignité. Toutefois, certains auteurs ont rapporté l'association de I'HL à divers processus malins (lymphome, leucémie, cancer du sein), sans apporter de preuve formelle quant à un lien entre ces deux entités [8]. De plus, si un tel lien existait, il pourrait être expliqué, par un effet de carcinogenèse induite par certains traitements de I'HL.

L'HL fait intervenir la prolifération de cellules apparentées aux phagocytes mononucléés ayant comme rôle principal la phagocytose et/ou la présentation d'antigène. Ces cellules sont identifiées par l'expression de la protéine $\mathrm{S} 100$ et de l'antigène CD1a à leur surface et par la présence des " granules de Birberck » intracytoplasmiques ou " corps $X$ » donnant, en microscopie électronique, un aspect classique en raquette de tennis ou en fermeture à glissière $[4,5]$.

Le granulome éosinophile de l'os reste longtemps asymptomatique. Lorsqu'il devient symptomatique, il se manifeste essentiellement par des douleurs osseuses, parfois par des déformations osseuses, des fractures pathologiques ou des signes neurologiques en rapport avec une compression médullaire ou radiculaire [6]. L'imagerie de la lésion osseuse se présente typiquement sous la forme d'une lacune arrondie, de taille variable, sans condensation périphérique $[5,6]$. Les lésions du rachis siègent habituellement au niveau du corps vertébral et épargnent le disque intervertébral. Elles peuvent être uniques, ou multiples, s'étendant aux vertèbres adjacentes ou éloignées. Au niveau mandibulaire, l'atteinte de l'os basilaire se manifeste dans la plupart des cas sous forme d'une lacune bien limitée, circulaire ou ovalaire, sans condensation osseuse réactionnelle, comme c'est le cas de notre patient. Au niveau de l'os alvéolaire, on retrouve plutôt des lacunes détruisant les septas intra-alvéolaires, réalisant ainsi des images en panier. Les dents paraissent suspendues dans le vide, on parle alors de dents flottantes. Les lésions crâniennes réalisent des images d'ostéolyse arrondies ou polycycliques, à limites nettes, souvent dépourvues de liseré périphérique. Les radiographies du squelette entier sont la principale investigation pour l'atteinte osseuse. La scintigraphie osseuse au Tc99m est moins sensible mais parfois complémentaire des clichés standards. La tomodensitométrie est utile au diagnostic topographique, à la surveillance de l'évolution et au suivi de la réponse au traitement. L'IRM est encore plus performante pour la visualisation des lésions et de leur extension à la médullaire et aux tissus 
voisins. Le granulome osseux solitaire ou multiple, sans atteinte viscérale, comme le cas de notre patient, reste une affection bénigne, spontanément résolutive le plus souvent [5].

Les facteurs de mauvais pronostic sont [9] :

- l'âge jeune au moment du diagnostic initial, inférieur à 2 ans ;

- le nombre élevé des localisations ;

- la rapidité d'évolution de la lésion ;

- l'atteinte des poumons, du foie, de la rate et de la moelle osseuse ;

- la mauvaise réponse au traitement initial.

Le traitement est encore mal codifié. En cas de granulome osseux simple ou de lésions osseuses peu nombreuses, comme c'est le cas de notre patient, la majorité des auteurs préconisent un simple traitement antalgique par l'indométacine, la pose d'un corset ou d'une minerve dans

\section{Conclusion}

L'histiocytose langerhansienne est une entité rare, de présentation clinique variable. Le granulome éosinophile est la forme la plus fréquente. Il atteint essentiellement l'os, en l'occurrence les os de l'extrémité cranio-faciale. Seule l'histologie permet de confirmer le diagnostic. L'atteinte osseuse uni- ou multifocale est souvent de bon pronostic. Le traitement un but antalgique. Un traitement chirurgical non agressif peut être envisagé, il consiste à faire un curetage avec une éventuelle ostéosynthèse au niveau des épiphyses des vertèbres ou des métaphyses des os longs, afin de réduire le risque fracturaire. Les complications neurologiques par extension vers la moelle épinière et les racines nerveuses peuvent être prévenues par une infiltration de corticoïdes dans la lésion à visée antiinflammatoire $[3,5,6]$. En cas d'atteinte osseuse multifocale avec de nombreuses localisations, le traitement fait appel à la chimiothérapie (vinblastine, etoposide, vincristine, cyclophosphamide...) seule ou associée à une corticothérapie. La radiothérapie doit être de principe évitée en raison des risques de cancer secondaire. Une nouvelle stratégie thérapeutique est en cours d'étude, elle utilise des anticorps monoclonaux anti-CD1a.

est controversé. Toutefois, pour les lésions osseuses, uniques ou peu nombreuses, beaucoup d'auteurs optent soit pour une abstention thérapeutique, soit pour une chirurgie non agressive (curetage), et éventuellement une injection locale de corticoïdes ou d'un traitement anti-inflammatoire en cas de douleur.

\section{Bibliographie}

1. Nguyen $\mathrm{KH}$, Tazi $\mathrm{A}$.

Histiocytose

Langerhansienne

de l'adulte.

Rev Prat 2006;56:1863-71.
2. Favara $B E$, Feller $A C$, Pauli M, et al. Contemporary classification of histiocytic disorders. The WHO committee on histiocytic/reticulum cell proliferations. Reclassification Working Group of the Histiocyte Society. 
Med Pediatr Oncol

1997;29(3):157-66.

3. Lichtenstein L, Jaffe HL. Eosinophilic granuloma of bone: with report of a case. Am J Pathol 1940;16(5):595-604.

4. Chu T, D' Angio J, Favara B, Ladisch S, Nesbit M, Pritchard J.

The Writing Group of The Histiocyte Society. Histiocytosis syndromes in children.

Lancet 1987;1(8526):208-9.

5. Feugier $P$, Guergi $A$, Nafissi S, Loderlin P. Granulomes éosinophiles uni et multifocaux de l'adulte.

Approches diagnostiques et thérapeutiques. A propos de trois observations. Rev Med Interne 1996; 17:924-8.

6. Ghanem I, Checrallah A, Kharrat K, Dagher F. Histiocytose à cellules de Langerhans. Appareil locomoteur. Encycl Méd Chir. Elsevier SAS, Paris, 2001;14-776:1-14.

7. Wilman $\mathrm{CL}$, Busque $\mathrm{L}$, Griffith BB , Favara BE, McClain KL, Duncan MH, Gilliland DG.

Langerhans'-cell histiocytosis (histiocytosis X). A clonal proliferative disease.

N Engl J Med

1994;331(5):154-60.

8. Egeler RM, Neglia JP, Puccetti DM, Brennan CA, Nesbit ME.

Association of Langerhans cell histiocytosis with malignant neoplasms. Cancer 1993;71(3):865-73.

9. Lallemant $B$, Fayoux $P$, Nelken B, Leroy $\mathrm{X}$, Vaneecloo FM. Du diagnostic à la prise en charge des localisations ORL de l'histiocytose Langerhansienne chez l'enfant.

Ann Otolaryngol Chir Cervicofac 2003;120(1):30-9.

\section{SUMMARY}

\section{Multifical Langerhans cell histiocytosis of bone:}

\section{a case report}

Hicham SABANI, M. Karim EL KHATIB, Jalal HAMAMA, Mohammed NASSIH, Abdelkader RZIN, Youssef ZOUBIR, Mohammed OUKABLI, Abderrahmane ALBOUZIDI

\section{Keywords \\ - Langerhans cell histiocytosis \\ - eosinophilic granuloma - multiple bone lesions}

Langerhans cell histiocytosis is a non-malignant proliferative disease that involves dendritic Langerhans cells normally found in the skin and mucous membranes located in the interface with external environment. Langerhans cell histiocytosis is a rare disease. Only histology can confirm the diagnosis. The treatment is still controversial.

We report a case of a young man with multifocal osseous histiocytosis $\mathrm{X}$, involving initially the rachis, and secondarily the mandible and the skull. 